DOI: $10.20472 /$ IAC.2019.049.033

\author{
KEITA NISHIO \\ Aoyama Gakuin University, Japan \\ TAKASHI KABURAGI \\ International Christian University, Japan \\ SATOSHI KUMAGAI \\ Aoyama Gakuin University, Japan \\ TOSHIYUKI MATSUMOTO \\ Aoyama Gakuin University, Japan \\ YOSUKE KURIHARA \\ Aoyama Gakuin University, Japan
}

\title{
NON-INVASIVE METHOD FOR ESTIMATING BLOOD GLUCOSE LEVEL UTILIZING HARMONIC DISTORTION OF PULSE PRESSURE
}

\begin{abstract}
:
In this paper, a non-invasive method for estimating blood glucose level is proposed. We investigate blood viscosity and harmonic distortion using a model with the assumption that high blood viscosity caused by high blood glucose level generates turbulence in blood flow. This consequently leads to the distortion of fundamental frequency of pulsation. Therefore, the harmonic components appear owing to the pressure on the vascular wall. Using the proposed non-invasive method, we calculate the amplitude of the harmonic components. The blood glucose level is estimated with a single regression model whose explanatory variable is the calculated harmonic component. Through validation experiment with 33 measured data, the third harmonic component achieved the highest estimation accuracy with $60 \%$ of the data meeting the ISO criteria.
\end{abstract}

\section{Keywords:}

non-invasive, blood glucose level, regression model, diabetes, harmonic distortion, blood pressure monitor, Fast Fourier transform

JEL Classification: I19 


\section{Introduction}

According to the World Health Organization (WHO), the number of diabetes patients is assumed to be over 422 million [1]. Moreover, the International Diabetes Federation reports the prevalence of diabetes is $8.8 \%$ and will reach $9.9 \%$ by 2045 [2]. The current method for controlling blood glucose level is through insulin injection. Before injecting insulin, a patient needs to take a blood sample to measure their blood glucose level. To do so, a puncture needle is typically used. However, blood withdrawal with a puncture needle is accompanied by some problems, such as scars left by the puncture needle, stress owing to the pain and a risk of infection. Therefore, a non-invasive method for measuring the blood glucose level is required.

Non-invasive methods for estimating blood glucose level have been previously proposed in the literatures [3]-[5]. In the literatures [3], [4], the property of absorbance of blood under the skin is utilized for estimating. This method achieves high-precision estimation; however, they are not suitable for day-to-day monitoring because the measurement instruments are huge and costly. In the literature [5], the blood glucose level is estimated by analyzing a teardrop. Although the teardrop analyzing does not cost too much, the estimation precision is relatively low.

In this paper, we propose a non-invasive blood glucose level estimation method, which uses an arm-cuff blood pressure monitor.

\section{Proposed Method}

The proposed method consists of two parts: the model part and the signal processing part. The model part represents a diabetes patient's blood flow when they are measuring their blood pressure using an arm-cuff blood pressure monitor. In the other part, blood glucose level is estimated using signal processing based on the model.

\section{A. Modelling of blood viscosity and harmonic distortion}

First, the blood viscosity is assumed to be low if the blood glucose level is low. Conversely, the blood viscosity is assumed to be high if the blood glucose level is high.

When a patient measures the blood pressure using an arm-cuff blood pressure monitor, the cuff shrinks and compresses the blood vessel, thereby stopping the blood flow of the arm. When the cuff loosens and the blood vessel expands, blood starts to flow again. The blood flow after the cuff loosens is influenced by blood viscosity. Figure 1 shows the modelling of blood viscosity and harmonic distortion. If the blood viscosity is low, the blood flows smoothly when the cuff loosens. If the blood viscosity is high, the blood flows less smoothly compared to the case of low blood viscosity. Here, we consider the pressure on the vascular wall due to a single pulsation. In the case of low blood viscosity, pressure fluctuation with the fundamental frequency of pulsation occurs on the vascular wall. Whereas, in the case of high blood viscosity, turbulence occurs, leading to vibrations on the vascular wall. Subsequently, the fundamental frequency of pulsation is distorted, and the harmonic components appear. The proportion of the harmonic components 
to the fundamental frequency is related to the blood viscosity. This leads to large harmonic components of the pressure on the vascular wall.

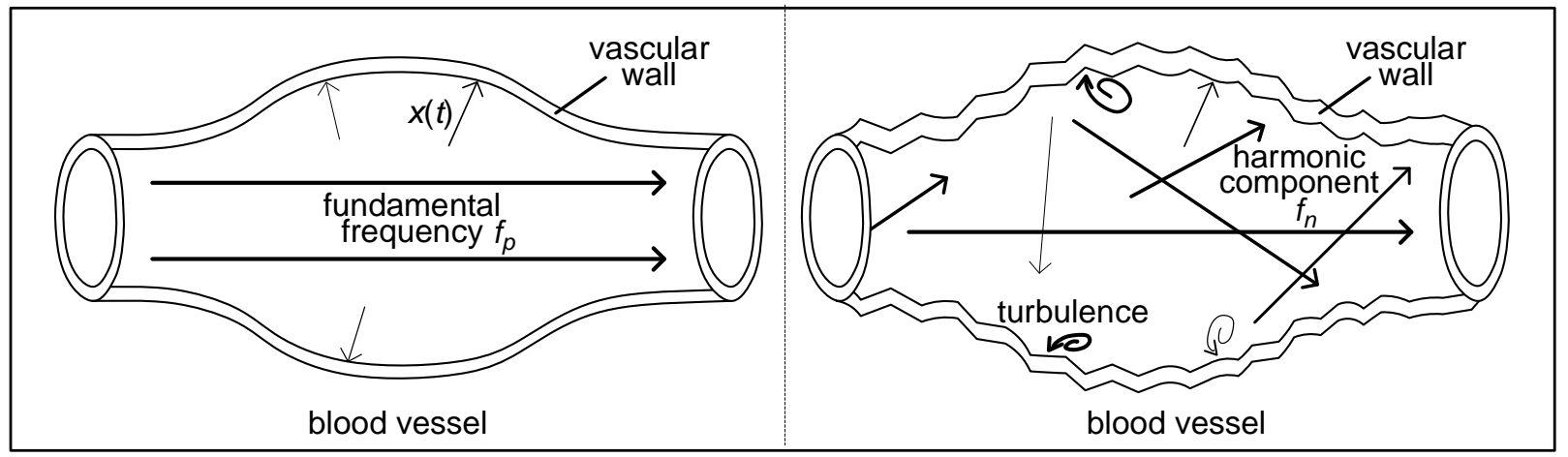

(a)Low blood viscosity

(b)High blood viscosity

Fig.1 Modelling of blood viscosity and harmonic distortion of pressure on vascular wall

Given that the fundamental frequency of the pulse pressure component is $f_{p}$, the $n$th harmonic component appears at frequency $f_{n}(n=1,2, \ldots)$, as shown in Eq.(1). Furthermore, if $a_{f_{n}}$ is the spectrum of the $n$th harmonic component, then

$$
f_{n}=(n+1) f_{p} \#(1)
$$

Here, $x(t)$ is the pressure on vascular wall, where $t$ is the continuous time. $x(t)$ is expressed as the summation of several harmonic components, as shown in Eq.(2).

$$
x(t)=\sum_{n=0}^{\infty} \sin \left[2 \pi(n+1) f_{p} t\right] \#(2)
$$

Because $a_{f n}$ is related to a blood glucose level, the estimated blood glucose level $\widehat{B}$ is expressed as a function of $a_{t_{n}}$ as shown in Eq.(3).

$$
\widehat{B}=f\left\{a_{2 f_{p}}, a_{3 f_{p}}, a_{4 f_{p}}, \ldots\right\} \#(3)
$$

\section{B. Signal Processing}

Let us take $y(t)$ as the output signal from the pressure sensor. It is the sum of the noise components and the pressure on vascular wall $x(t)$. This output signal $y(t)$ is $\mathrm{A} / \mathrm{D}$ converted with a sampling frequency $f_{s}$ and transferred to a computer as a digital signal $y(k)(k=1,2, \ldots, M)$, where $k$ is the discrete time step with sampling frequency $f_{s}$, and $N$ is the number of data points. $N$ is different for each data because the measurement time varies for each measurement. However, each data needs to have the same number of data points such that all data have the same frequency resolution. Accordingly, data with fixed $N_{e}$ data points is extracted from each $y(k)$. Fast Fourier transform (FFT) is applied to the extracted data and an amplitude spectrum $a_{f}(f=0, \Delta f$, 
$\left.2 \Delta f, \ldots, f_{s}\right)$ is obtained, where the frequency resolution $\Delta f$ is $\Delta f=f_{s} / N_{e}$. Here, the fundamental frequency $f_{p}$ is determined as the discrete frequency having the largest spectrum in the range of 0.8-6.4 Hz. Next, the discrete frequency that corresponds to the $n$th harmonic component is calculated based on Eq.(2). To evaluate the size of $n$th harmonic component, a difference between the spectra around the $n$th harmonic component is calculated. However, these spectra can be affected by the presence of noise components. Moreover, as the frequency is discrete, $f_{n}$ is not representative of the exact integer multiple frequency of the $n$th harmonic component. For these reasons, we calculate the differences between two adjacent spectra for $m$ discrete frequencies above and below $f_{n}$. To evaluate the magnitude of the differences, the differences are converted into their absolute values. We consider $S_{n}$ to be the sum of the absolute values around $f_{n}$. It can be calculated using Eq.(4) and represents the amount by which the $n$th harmonic component influences $y(t)$. In the case of low blood glucose level, $S_{n}$ is expected to be small. By contrast, in the case of high blood glucose level, $S_{n}$ is expected to be large.

$$
S_{n}=\sum_{i=-(m-1)}^{m}\left|a_{f_{n}+i \Delta f}-a_{f_{n}+(i-1) \Delta f}\right| \#(4)
$$

Finally, the function in Eq.(3) is determined by a single regression model whose explanatory variable is $S_{n}$.

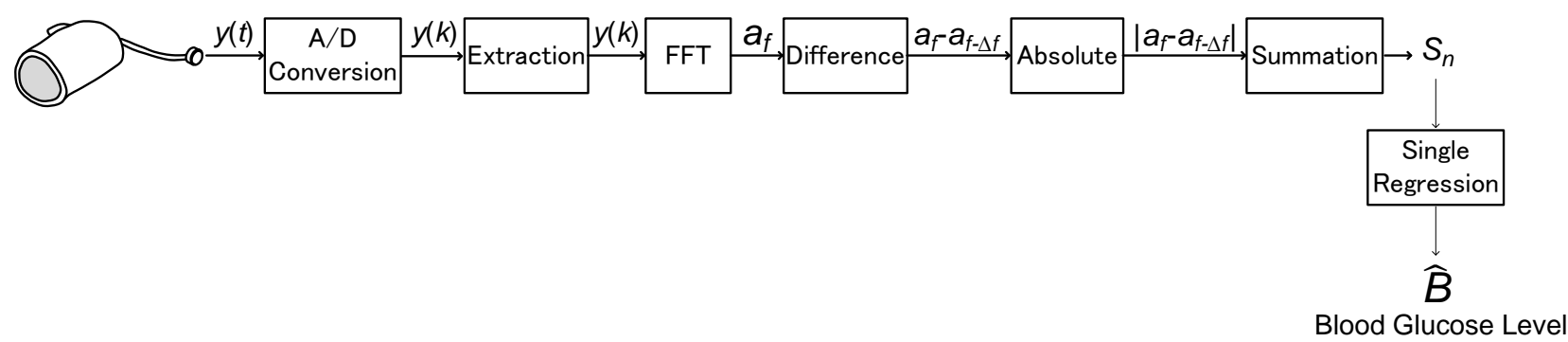

Fig.2 Signal processing for estimating blood glucose level.

\section{Experiment}

To evaluate the efficiency of the proposed method, we conducted the validation experiment. This section describes the experimental system, experimental procedure, and evaluation criteria.

\section{A. Experimental System}

As the sensing device to measure the pulse pressure, the arm-cuff blood pressure monitor HME7120 (OMRON HEALTHCARE Co., Ltd.) was used. The tube connecting the cuff to the monitor was bifurcated and Electret Condenser Microphone EM114(PRIMO CO., LTD) was attached to one of the bifurcated tubes. The output signal from EM114 was amplified by a low-frequency amplifier MX4836 (PRIMO., Co. Ltd) with a maximum gain of 40.4dB. The amplified signal was A/D converted with AIO-160802AY-USB (Contec Co., Ltd.) with the sampling frequency $f_{s}$ 
$=1000 \mathrm{~Hz}$ and transmitted into a computer. The imported data $y(k)$ were then inputted. Accu-Chek® Mobile (F. Hoffmann-La Roche, Ltd.) was used to measure the true blood glucose level.

\section{B. Experimental Procedure}

The participants for the experiment were two males, one in his thirties and the other in his forties. The total number of obtained measurements was 33. Before the measurement, each participant was asked to measure their true blood glucose level using Accu- Chek® Mobile. Next, they were asked to wear the arm-cuff blood pressure monitor HME-7120 on their arms and measure the pressure change $y(t)$. The digital signal $y(k)$ was then obtained in parallel with the measurement. $y(k)$ is analyzed by the proposed method, where $m=3$ and $N_{e}=10000$. Here, we validate the following four patterns as the extracted interval with $N_{e}$ points: the first $10 \mathrm{~s}(k=1,2, \ldots, 10000)$, the intermediate $10 \mathrm{~s}(k=10001,10002, \ldots, 20000)$, the last $10 \mathrm{~s}(k=N-9999, N-9998, \ldots, N)$ and the overall data $(k=1,2, \ldots, M)$.

\section{Evaluation Criteria}

To evaluate if the estimated glucose level is valid, we use the criteria established by the International Organization for Standardization (ISO). We consider e to be the error between true blood glucose level $B$ and estimated blood glucose level $\widehat{B}$. The ISO criteria are shown in Eq.(5). We applied leave-one-data-out cross validation to the measured data. A portion of the data was selected as test data. The remaining was used as training data to train a single regression model. Applying the test data to the trained regression model, the blood glucose levels of the test data was estimated. The proposed method was evaluated using the Accuracy defined in Eq.(6), where num is the total number of all data points and num' is the number of datapoints that meet the ISO criteria. Accuracy was calculated for 40 combinations because the validation was done up to the tenth harmonic component and the four patterns of the extracted interval.

$$
\begin{array}{cc}
\left\{\begin{array}{l}
e \leq \pm 15 \mathrm{mg} / \mathrm{dL} \\
\frac{e}{B} \times 100 \leq \pm 20 \%
\end{array}\right. & (B<75 \mathrm{mg} / \mathrm{dL}) \\
\text { Accuracy }= & \frac{\text { num }^{\prime}}{\text { num }} \#(6)
\end{array}
$$

\section{Results}

This section illustrates the typical measured spectra and an estimation result.

\section{A. Typical measured spectra}

Figure 3 shows the typical spectra obtained by the FFT of the overall data in a (a) low blood glucose level, (b) moderate blood glucose level, and (c) high blood glucose level. The true blood glucose level of (a) low blood glucose level is $93 \mathrm{mg} / \mathrm{dL}$. The spectra of the harmonic components 
are not so large, although the second harmonic has a little larger spectrum than the other harmonics in this data. Concerning the data of (b) moderate blood glucose level, the true blood glucose level is $110 \mathrm{mg} / \mathrm{dL}$. The spectra of the harmonic components are not significantly large, but slightly larger than those of a (a) low blood glucose level. Concerning the data of (c) high blood glucose level, the true blood glucose level was $160 \mathrm{mg} / \mathrm{dL}$. The spectra of the harmonic components were the largest among the three patterns of glucose levels.

These results indicate that $a_{f_{n}}$ of the harmonic components are large, owing to the high blood glucose level.

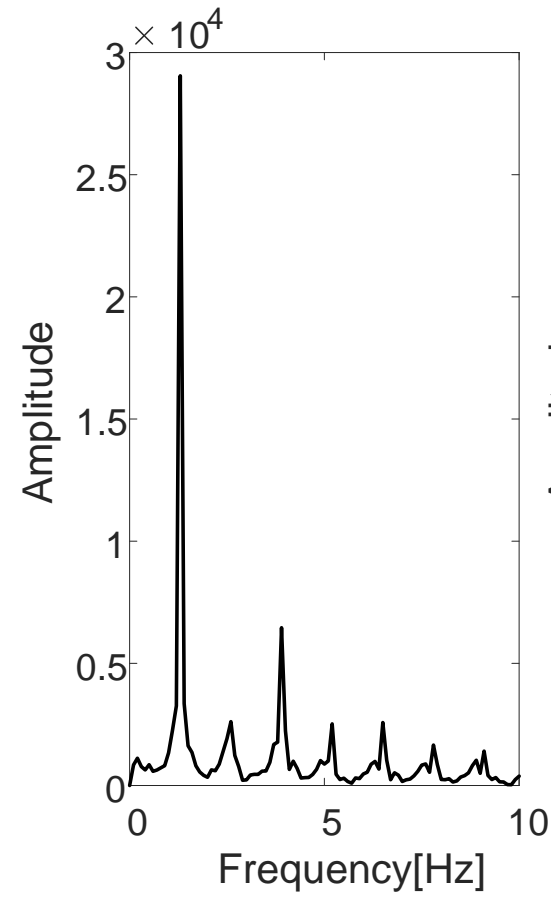

(a) Low glucose level

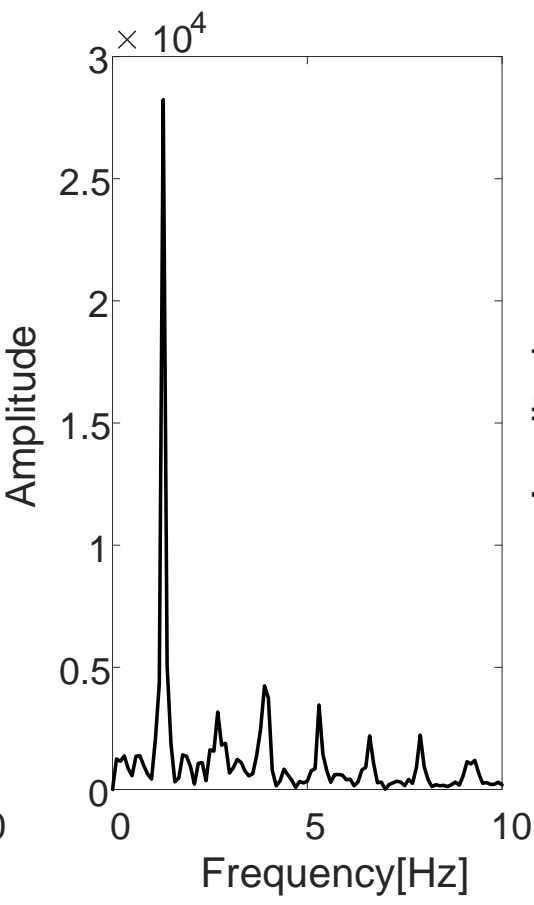

(b) Moderate glucose level

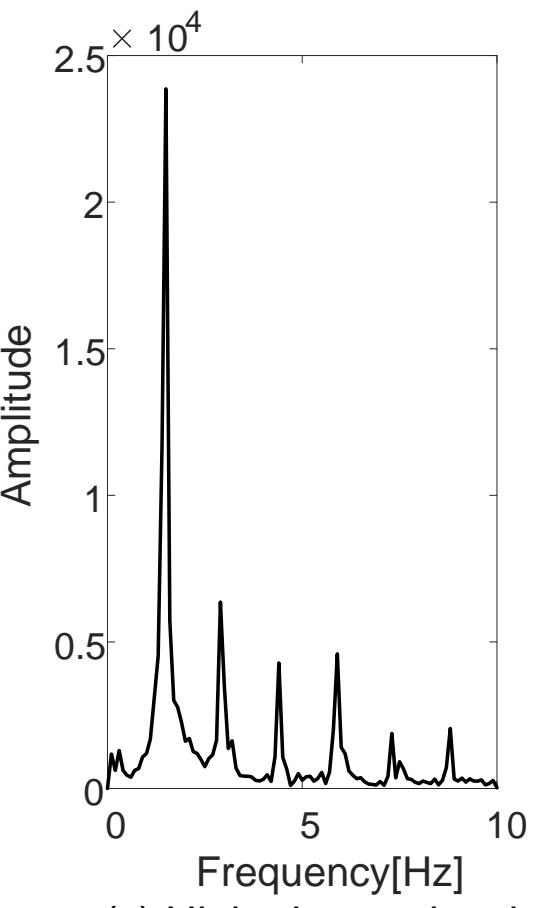

(c) High glucose level

Fig.3 Typical spectra of (a) Low glucose level, (b) Moderate glucose level and (c) High glucose level.

\section{B. Estimation Results}

Table. 1 shows the Accuracy for each combination of harmonic components and extracted intervals. Out of all the combinations, the third harmonic component of the last $10 \mathrm{~s}$ achieved the highest Accuracy of 0.60 . In terms of an average of each harmonic component among all the extracted intervals, the third and the fifth harmonic components together yielded the highest Accuracy of 0.47 . 
Table.1 Accuracy of 40 combinations of harmonic components and extracted intervals

\begin{tabular}{|c|c|c|c|c|c|c|c|c|c|c|c|}
\cline { 2 - 12 } \multicolumn{1}{c|}{} & \multicolumn{10}{c|}{ nth harmonic component } & \multicolumn{1}{c|}{} \\
\cline { 2 - 13 } \multicolumn{1}{c|}{} & 1 & 2 & 3 & 4 & 5 & 6 & 7 & 8 & 9 & 10 & Average \\
\hline First & 0.42 & 0.39 & 0.36 & 0.42 & 0.45 & 0.51 & 0.45 & 0.45 & 0.42 & 0.36 & 0.42 \\
\hline Middle & 0.45 & 0.54 & 0.48 & 0.42 & 0.54 & 0.42 & 0.39 & 0.42 & 0.42 & 0.45 & 0.45 \\
\hline Last & 0.45 & 0.42 & $\mathbf{0 . 6 0}$ & 0.45 & 0.48 & 0.39 & 0.45 & 0.45 & 0.45 & 0.39 & 0.45 \\
\hline Overall & 0.39 & 0.42 & 0.45 & 0.39 & 0.39 & 0.48 & 0.45 & 0.39 & 0.39 & 0.45 & 0.42 \\
\hline Average & 0.43 & 0.44 & 0.47 & 0.42 & 0.47 & 0.45 & 0.44 & 0.43 & 0.42 & 0.41 & \\
\end{tabular}

\section{Discussion}

As stated in the previous section, $a_{f n}$ of the harmonic components are large in the case of high blood glucose level, which validates the model of blood viscosity and harmonic distortion. However, the estimation of blood glucose level was done using a single regression model whose explanatory variable was $S_{n}$. As future work, we plan to consider multiple regression analysis for the estimation of blood glucose level.

\section{References}

[1] Diabetes Fact Sheet, World Health Organization (WHO), Oct. 2018. (https://www.who.int/news-room/fact-sheets/detail/diabetes)

[2] IDF Diabetes Atlas Eighth Edition 2017, International Diabetes Federation (IDF), 2017. (https://diabetesatlas.org/IDF_Diabetes_Atlas_8e_interactive_EN/)

[3] Andrew J. Berger, T. Koo and I. Itzkan, G. Horowitz, M. Feld, "Multicomponent blood analysis by near-infrared Raman spectroscopy," APPLIED OPTICS, Vol.38, No.13, pp.2916-2926, 1999.

[4] Andrew J. Berger, I. Itzkan and M. Feld, "Feasibility of measuring blood glucose concentration by near-infrared Raman spectroscopy," Spectrochimica Acta Part A: Molecular and Biomolecular Spectroscopy, vol.53, issue.2, pp.287-292, Feb.1997.

[5] Ming Xing Chu, K. Miyajima, D. Takahashi, T. Arakawa, K. Sano, S. Sawada, H. Kudo, Y. Iwasaki, K. Akiyoshi, M. Mochizuki and Kohji Mitsubayashi, "Soft contact lens biosensor for in situ monitoring of tear glucose as non-invasive blood sugar assessment," Talanta, vol.83, no.3, pp.960-965, 2 